\title{
QUELQUES RĒGLES POUR LA CULTURE DES PEUPLIERS NOIRS EN PÉPINIËRE ET LEUR MISE EN PLACE
}

\section{Qualités de la Pépinière}

Une pépinière parfaitement adaptée à la production de plants forestiers, résineux ou feuillus, ne peut bien souvent être utilisée comme pépinière de peupliers: les premiers sont destinés à des sols relativement pauvres et secs, et, même pour les espèces dites à croissance rapide, n'atteignent qu'un faible développement annuel; les seconds sont destinés à des sols riches et bien alimentés en eau et leur croissance annuelle est considérable.

Le sol de la pépinière de peupliers doit être riche, profond et frais. Il ne doit pas être trop compact, et il est préférable qu'il soit léger si l'alimentation des plants peut être régulièrement et largement assurée par arrosage ou mieux par irrigation. Un sol d'alluvions assez fines, homogène sur une profondeur minimum de $0,60 \mathrm{~m}$, neutre ou même calcaire, convient parfaitement. En outre, ce sol doit avoir été profondément défoncé et être bien ameubli ; on y incorpore des engrais pas trop riches en azote.

Bien entendu, les sarclages doivent faire disparaitre les mauvaises herbes favorisées par la richesse et l'humidité du sol et dont la concurrence nuit beaucoup aux peupliers.

Une pépinière de peupliers mal installée ou mal préparée donne des déboires aussi spectaculaires que la rétussite d'une bonne pépinière; nous avons en $195^{2}$ constaté dans deux d'entre elles distantes de moins de $100 \mathrm{~km}$, avec des boutures du mème lot, les résultats suivants:

Sol profond, bien travaillé et irrigué:

Pourcentage de reprise $\ldots \ldots \ldots \ldots \ldots \ldots .88 \%$

Hauteur moyenne $\ldots \ldots \ldots \ldots \ldots \ldots \ldots .2,25 \mathrm{~m}$

Sol sec, travaillé superficiellement à la pioche et arrosé :

Pourcentage de reprise $\ldots \ldots \ldots \ldots \ldots \ldots$..... $45 \%$

Hauteur moyenne $. . . \ldots \ldots \ldots \ldots \ldots \ldots .0,56 \mathrm{~m}$ 


\section{Choix des boutures}

Les boutures doivent être entièrement constituées de bois formé durant la précédente saison de végétation. On les prélève soit dans la cime d'arbres jeunes, vigoureux et identifiés avec certitude, soit par élagage ou recépage (voir ci-dessous) des plants élevés dans la pépinière, soit sur des pieds-mères traités en têtards, en vue de la production de boutures (plants de 2 ans recépés à une vingtaine de centimètres de hauteur au-dessus du sol et espacés de $0,50 \mathrm{~m}$ sur des lignes distantes de I $\mathrm{m}$ en sol travaillé et fumé).

On les prélève pendant l'arrêt de la végétation (et on les met en jauge dans le sable, la pointe en bas) ou mieux on les récoltie peu de temps avant leur mise en place.

Ces boutures doivent avoir au moins la grosseur d'un crayon, diamètre minimum : $8 \mathrm{~mm}$, et une longueur de 0,20 à $0,35 \mathrm{~m}$.

Elles doivent être saines, droites, ni blessées ni attaquées par un parasite animal ou végétal et leurs bourgeons doivent être normalement développés.

\section{Bouturage et culture}

Les boutures sont enfoncées verticalement dans le sol bien travaillé ou placées côte à côte dans une tranchée à bord abrupt qui est comblée ensuite: l'extrémité supérieure que la direction des bourgeons permet de reconnaitre sans hésitation, ne doit pas dépasser le sol; elle doit affleurer la surface.

L'espacement des plants sur les lignes et entre les lignes dépend de la méthode de culture choisie. Cependant l'écartement des lignes doit toujours être suffisant pour permettre le passage des instruments de culture mécanique en usage dans la pépinière.

Il est d'ailleurs nécessaire de préciser que la circulation entre les lignes est plus ou moins facile suivant qu'on cultive un clone à tige droite (type ( robusta )) ou au contraire flexueuse (type " virginie $)$ ).

a) Mise en place sans transplantation ultérieure.

C'est la méthode la plus simple et la plus couramment utilisée en France.

Les boutures sont plantées à 0,30 à $0,50 \mathrm{~m}$ les unes des autres sur des lignes distantes au minimum de $\mathrm{I} \mathrm{m}$ et au maximum de 2 mètres.

L'espacement minimum sera adopté lorsque les plants ne resteront qu'une année en place (production de plants de $\mathrm{O}-\mathrm{I}$ ), ce qui est exceptionnel et peu souhaitable.

L'espacement maximum le sera lorsque les plants resteront trois années en place (production de plants de o-3).

On laisse une seule pousse se développer sur chaque bouture et 
on la taille légèrement au cours de l'année pour lui donner une bonne forme.

Lorsque les plants ont atteint 1'âge et la dimension choisis pour leur plantation, ils sont extraits et triés avec élimination des sujets défectueux.

Avantages: très grande simplicité; risque d'erreurs très réduit; possibilité d'extraire les plants à des âges variés (O-I, O-2 ou 0-3) suivant leur développement et l'importance des besoins.

Main d'œuvre réduite.

Inconvénients: occupation imparfaite du terrain surtout lorsque le pourcentage de reprise des boutures n'a pas été très élevé; aucune élimination des plants insuffisants avant l'extraction finale; tentation de prélever les plants suffisamment forts et d'employer ultérieurement à un âge plus avancé des sujets que leur développement médiocre aurait dî̀ faire éliminer; production de boutures réduite.

Cette méthode doit être employée ou conservée dans les pépinières cultivant plusieurs clones et où la main-d'œuvre insuffisamment différenciée risquerait de commettre des erreurs dans les manipulations nécessitées par la méthode c.

b) Même méthode avec recépage.

Elle est employée surtout lorsque la croissance en hauteur a été trop rapide la première année ou, au contraire, a été irrégulière et défectueuse. Elle consiste à recéper tous les plants à quelques centimètres au-dessus du sol avant le départ de la végétation de la $2^{\mathbf{e}}$ année.

Les pousses ainsi coupées fournissent de bonnes boutures. Les plants obtenus ont une tige qui a un an de moins que le système radiculaire; ils sont généralement moins allongés, mais de diamètre plus fort que sans recépage.

Cette méthode est intermédiaire entre a et $c$.

c) Méthode avec recépage et transplantation.

C'est une méthode qui est utilisée en Italie par le Professeur PicCAROLO.

Les boutures sont mises en place sur des lignes distantes de I m à $\mathrm{O}, \mathrm{IO}$ à $\mathrm{O}, \mathrm{I} 5$ les unes des autres.

$\mathrm{Au}$ bout d'un an elles sont extraites et recépées à 2 ou 3 bourgeons au-dessus du collet. La pousse est utilisée pour faire des boutures.

Les racines des petites souches sont taillées très court, puis elles sont mises en place à $0,50 \mathrm{~m}$ les unes des autres sur des lignes distantes de 2 mètres. 
Les plants sont extraits et utilisés deux ans plus tard: leur qualification est alors la suivante " O-I-2 ".

Avantages: très bonne utilisation du terrain qu'on peut encore améliorer en plaçant les boutures sur des lignes temporaires entre celles où viennent d'être installées les souches.

Production abondante de boutures de qualité,

Elimination de tous les sujets défectueux et en particulier de ceux dont le système radiculaire est insuffisant.

Production de plants forts, pas trop allongés et bien racinés.

Inconvénients: nécessité d'une main-d'œuvre importante et expérimentée.

Risque de confusions entre les clones lorsque plusieurs sont cultivés dans la pépinière.

Nous conseillons cette méthode lorsque la pépinière ne produit qu'un clone ou lorsqu'elle a un personnel suffisamment qualifié pour que les erreurs soient peu à craindre.

\section{Contrôle sanitaire}

On peut rarement lutter avec efficacité contre les ennemis des arbres dans les peuplements forestiers, même lorsque comme c'est généralement le cas pour les peupliers, ils sont faciles d'accès et plantés en alignement ou en massifs clairs et peu étendus. Par contre, il est toujours aisé de limiter le développement des insectes ou des champignons dans la pépinière: cette lutte a d'autant plus d'importance que la distribution de plants infectés diffuse largement les parasites.

Une étude détaillée des ennemis des peupliers sortirait du caclre de cette note; notre but est simplement d'attirer l'attention sur les principaux et d'indiquer les moyens de les combattre.

Nous les grouperons d'après la nature de l'organe attaqué :

I Parasites des feuilles. - A côté de certaines larves mineuses peu dangereuses, les larves et même les adultes de nombreux insectes dévorent les feuilles de façon plus ou moins complète (Chrysomèles. diverses Chenilles, Altises, etc...).

Quelques charançons et pucerons les déforment.

Quant aux champignons, certains causent des cloques de couleur vive, mais les plus fréquents sont les rouilles qui ne se développent qu'à la fin de l'été et accélèrent seulement la chute des feuilles.

S'ils attirent aisément l'attention et peuvent affaiblir les plants, les parasites des feuilles sont rarement très dangereux. Il est cependant indispensable de limiter leur développement.

$2^{\circ}$ Parasites des tiges et des rameaux. - Ils sont beaucoup plus à craindre. 
La Petite Saperde pond sur les rameaux ou les parties supérieures des tiges dont le diamètre est généralement inférieur à I $5 \mathrm{~mm}$; la présence de sa larve qui se développe à l'intérieur du bois, est décelée par une excroissance plus ou moins globuleuse. La tige ainsi déformée devient cassante.

La Grande Saperde, qui creuse ses galeries dans la partie inférieure des tiges de plus forte dimension, est plus rare dans les pépinières. (Il en est de même de quelques autres insectes mineurs Cossus, Sésies, etc...).

Un très dangereux champignon, Dotichiza populea, détermine une nécrose progressive des tiges ou des rameaux et souvent leur mort par annélation circulaire: cette maladie attaque généralement des sujets affaiblis et son extension est fréquente lors de la crise de transplantation. Il est donc indispensable que la pépinière ne soit pas un foyer pour sa diffusion.

Les bons soins donnés à la pépinière réduisent l'attaque du Dotichiza, mais n'ont guère d'influence sur les autres parasites. On peut limiter leurs dégâts par des mesures d'hygiène: couper et brûler les rameaux attaqués par la Petite Saperde ou le Dotichiza, rassembler et brûler les feuilles tombées précocement à la suite d'une attaque de Rouille; capturer les insectes parfaits (Chrysomèles) ou même détruire les larves par l'introduction d'un produit chimique dans leurs galeries.

Ces méthodes sont recommandables mais insuffisantes: seuls des traitements chimiques systématiques et régulièrement répétés élimineront la majorité des parasites et empêcheront leur extension. Sauf le cas d'une attaque particulièrement virulente qui devrait déclencher une action immédiate, on interviendra périodiquement par pulvérisation d'une solution polyvalente par exemple les I 5 mai, I 5 juin et fin juillet.

La bouillie bordelaise additionnée de D.D.T. convient parfaitement ainsi que certains produits spécialement préparés pour être associés: Rhodiacuivre I $\mathrm{kg}$ et Pacol spécial (I) 0,7 1. pour Ioo litres d'eau.

\section{Contrôle variétal dans la pépinière}

La pépinière doit produire exclusivement des plants exactement identifiés et dénommés et appartenant à des clones homologués par l'arrêté du 3 I octobre 1950.

Elle doit donc à l'origine être approvisionnée en boutures correspondant à ces exigences, mais ensuite il est indispensable qu'aucune erreur ne mélange les clones. De grandes précautions devront être prises lors des transplantations, des expéditions et de la récolte des boutures.

(I) Le principe actif du Pacol est un toxique violent; ce produit doit donc être utilisé avec précaution. 
En effet, si certains types (P. " carolin » et « angulata » et à un moindre degré, P. "( robusta 》) sont aisément distingués à tout âge, la plupart ne peuvent l'être pratiquement qu'à certaines époques de l'année.

Pour éviter les mélanges, on doit séparer nettement les clones dans la pépinière: en particulier une ligne commencée avec un clone ne doit jamais être achevée avec un autre.

Une deuxième prěcaution pour réduire les erreurs à l'arrachage consiste à marquer les tiges à la peinture, de couleurs conventionnelles, à $\mathrm{I} \mathrm{m}$ de hauteur environ avant leur extraction: ceci a l'avantage de permettre l'élimination des sujets insuffisants ou tarés qui ne seront pas marqués non plus que ceux dont l'exactitude est douteuse.

La destruction de tous les plants non marqués sera strictement prescrite et contrôlée.

Les tiges d'un an utilisées pour faire des boutures (méthodes b et c) pourront être marquées de la même façon avant recépage pour éviter les erreurs.

Au cours de la saison de végétation et particulièrement au printemps (pour les plants commençant leur deuxième année) on1 à l'auttomne, un bon observateur peut déceler les plants aberrants existants dans les carrés de la pépinière : ils seront immédiatement éliminés.

A titre d'information, nous indiquons ci-après les couleurs conventionnelles adoptés dang les Populetums:

Populus deltoides:

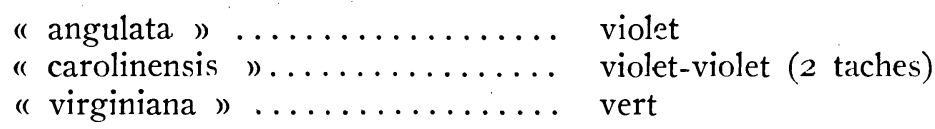

Populus euramericana :

" regenerata »............. jaune

" robusta $" . . . . \ldots \ldots \ldots \ldots . .$. rouge

" serotina de Champagne "...... blanc

" serotina du Poitou " .......... blanc-blanc (2 taches)

\section{Préparation des plants et choix}

Nous avons dit que préalablement à l'arrachage les plants " marchands " devraient être marqués de façon à ce qu'ils soient seuls livrés pour être plantés.

Ils seront ensuite élagués complètement (ce qui fournit des boutures), car la réduction de l'importance de la cime facilite la reprise; en aucun cas la tige principale ne sera raccourcie.

Les racines seront légèrement taillées: en particulier on rafrầ- 
chira la section de celles qui auront été endommagées à l'arrachage.

Lorsqu'on peut planter en " plançons ) (terrain très bien alimenté en eau) on coupera la tige au-dessus des racines au niveau du collet.

Dans tous les cas l'expérience prouve qu'il y a intérêt à utiliser des plants suffisamment forts: il semble qu'ils disposent de réserves plus importantes qui leur permettent de mieux développer ou reconstituer leur système radiculaire. En outre, leur tige principale est moins vulnérable aux attaques des petites saperdes; ils sont moins sensibles au Dotichiza et souffrent moins de la concurrence des plantes adventices qu'ils dominent plus rapidement.

La tige ne doit pas avoir moins de 8-1o centimètres de circonférence à $\mathrm{I} \mathrm{m}$ du collet et une hauteur de $2,50 \mathrm{~m}$ est un minimum.

\section{Mise en place}

Les Peupliers ne réussissent que dans des sols où ils peuvent être bien alimentés en eau, mais où ils ne souffrent pas de l'asphyxie des racines. Cependant, on peut distinguer deux grandes catégories de terrains où les peupliers peuvent être plantés et entre lesquels existent tous les intermédiaires. Il s'agit dans tous les cas de terrains de vallées plus ou moins régulièrement inondés.

$\left.\mathrm{I}^{\circ}\right)$ Sols humides toute l'année (le plan d'eau descend rarement au-dessous de I $\mathrm{m}$ de profondeur). - Il sera souvent nécessaire de procéder à leur drainage ou tout au moins de curer les fossés ou ruisseaux existants.

S'ils sont trop argileux (plus de $30 \%$ ), ils ne doivent pas être plantés en peupliers.

S'ils sont tourbeux, la tourbe devra être mélangée aux couches sous-jacentes si elle est superficielle, travaillée et aérée plusieurs mois à l'avance si elle est très épaisse.

Sur ces sols, les plus fréquemment réservés aux Peupliers en France, ils peuvent être plantés avec leurs racines ou sous forme de plançons; le plant doit être fortement butté au pied pour permettre aux racines de se développer au moins partiellement au-dessus du niveau de l'eau; la buttte de $0,40 \mathrm{~m}$ en tous sens protège le plant contre les vents.

La bonne alimentation en eau dispense généralement d'un travail du sol autour des plants pour faciliter la reprise, mais il est nécessaire au cours de l'été, de faucher ou mieux de détruire au Rotavator la puissante végétation qui pourrait étouffer les plants et qui est très combustible à la fin de l'hiver.

La plantation d'Aune en sous-étage (I ooo plants au maximum à l'hectare) est très favorable.

$2^{\circ}$ Sols où le plan d'eau varie de plusieurs mètres au cours de l'année. - Ces terrains peuvent être plantés en peupliers s'ils sont 
homogènes en profondeur, cə qui permet l'ascension capillaire de l'eau. Ils sont inutilisables si une couche de sable grossier ou de gravier est interposée entre le niveau du sol et le plan d'eau.

Les peupliers doivent y être plantés avec leurs racines et leur reprise est assurée si le sol est travaillé et entretenu pendant les premières années.

Le buttage n'est pas indispensable car les trous de plantation seront très profonds jusqu'à $0,80 \mathrm{~m}$ de profondeur et les racines ne doivent pas se développer en surface.

On peut associer sur ces sols d'alluvions bien assainis les peupliers à une culture agricole.

Dans les deux cas, les peupliers seront plantés an maximum à $7 \times 7 \mathrm{~m}$, soit 200 à l'hectare.

\section{Entretien des plantations}

Les soins de fauchage en sol humide, de travail du sol lorsqu'il est sec, sont nécessaires pendant 3 à 5 ans.

Par contre, l'apport d'engrais complet à la dose de $0,500 \mathrm{~kg}$ à I $\mathrm{kg}$ par pied qui peut favoriser le démarrage semble avoir peu d'effet ultérieurement.

Ensuite, suivant les cas, on livrera le terrain au pâturage (le bétail causerait des dommages aux arbres plus jeunes) ou bien on laissera le sous-étage d'aunes former taillis.

$\mathrm{Au}$ cours de ces 3 à 5 premières années aucune taille ne devra être pratiquée; il est en effet indispensable que le jeune arbre dispose du maximum de rameaux feuillés pour se nourrir. Cependant. on devra toujours procéder au défourchage, car les doubles cimes déprécient beaucoup les peupliers.

Les élagages commenceront ensuite et dégageront d'abord le tiers de la hauteur (jüsqu'à Io ans), puis la moitié (de ro à I 5 ans) et enfin les deux tiers. Ils seront pratiqués à la scie rez tronc et à l'aide d'une échelle; le Gui sera enlevé à l'occasion de ces élagages qui ne devront sous aucun prétexte être pratiqués à l'aide de crampons occasionnant aux arbres des blessures graves et dépréciant beaucoup le bois.

Mars I953.

Jean Pourtet. 